\title{
Complexité de suites définies par des billards rationnels
}

Bulletin de la S. M. F., tome 123, no 2 (1995), p. 257-270

<http://www.numdam.org/item?id=BSMF_1995_123_2_257_0>

(C) Bulletin de la S. M. F., 1995, tous droits réservés.

L'accès aux archives de la revue «Bulletin de la S. M. F. » (http: //smf.emath.fr/Publications/Bulletin/Presentation.html) implique l'accord avec les conditions générales d'utilisation (http://www.numdam.org/ conditions). Toute utilisation commerciale ou impression systématique est constitutive d'une infraction pénale. Toute copie ou impression de ce fichier doit contenir la présente mention de copyright.

\section{Numdam}


Bull. Soc. math. France,

123, 1995, p. 257-270.

\title{
COMPLEXITÉ DE SUITES DÉFINIES
}

\section{PAR DES BILLARDS RATIONNELS}

PAR

PASCAL HUBERT $(*)$

\begin{abstract}
RÉSUMÉ. - Soit $P$ un polygone rationnel convexe, $k_{1} \pi / r, \ldots, k_{q} \pi / r$ les angles entre deux côtés consécutifs où $k_{1}, \ldots, k_{q}, r$ sont premiers dans leur ensemble. Nous considérons le problème de billard dans ce polygone et codons les trajectoires suivant les côtés qu'elles rencontrent. Nous montrons que, si la suite ainsi obtenue n'est pas périodique, sa complexité est donnée par la formule $p(n)=n(q-2) r+2 r$. Cette expression de la complexité est valable pour $n$ assez grand et est indépendante des conditions initiales du problème.

ABSTRACT. - Let $P$ be a convex rational polygon, $k_{1} \pi / r, \ldots, k_{q} \pi / r$ the interior angles $\left(k_{1}, \ldots, k_{q}, r\right.$ are coprime). Let us consider the billiard problem in this polygon. We code the trajectories according to the sides they meet. When the sequence so obtained is not periodic, we show that the complexity of this sequence is equal to $p(n)=n(q-2) r+2 r$. This formula is true for $n$ large enough and does not depend on the initial conditions.
\end{abstract}

\section{Introduction}

On connaît peu de résultats concernant les propriétés ergodiques ou celles liées au codage du flot du billard dans un polygone quelconque. Trouver un exemple de flot de billard dans un polygone qui soit ergodique reste actuellement un problème ouvert. Toutefois, KerckHOFF, MASUR et Smillie ont montré qu'il y a un $G_{\delta}$ dense de polygones pour lesquels le flot est ergodique ( $c f$. [KMS]). Par ailleurs, KATOK démontre que ce sont des systèmes d'entropie nulle $(c f .[\mathrm{K}])$. Certains cas particuliers sont tout de même bien connus, tant au niveau ergodique que du point de vue du codage.

(*) Texte reçu le 10 novembre 1993, révisé le 17 février 1994.

Pascal HubERT, Laboratoire de Mathématiques Discrètes, CNRS-UPR 9016 case 930, 163 avenue de Luminy, 13288 Marseille CEDEx 9, France.

Email : hubert@lmd.univ-mrs.fr.

Classification AMS : 58F 03, 05 A 15.

BULLETIN DE LA SOCIÉTÉ MATHÉMATIQUE DE FRANCE 0037-9484/1995/257/\$5.00

(C) Société mathématique de France 
Le problème de billard le plus simple est celui du billard carré. On peut coder une trajectoire d'un tel billard par une suite infinie $m$ sur l'alphabet $\{0,1\}$ selon les côtés qu'elle rencontre : les côtés verticaux sont codés par 0 et les côtés horizontaux par 1 . On sait, depuis les travaux de Hedlund et Morse ( $c f$. $[\mathrm{HM}]$ ), que - lorsque la pente de la droite d'incidence est irrationnelle - la complexité du mot $m$ vaut

$$
p(n)=n+1
$$

pour tout nombre entier $n$ et que c'est la complexité minimale d'une suite non ultimement périodique. Réciproquement, toute suite dont la complexité est égale à $n+1$, pour tout nombre entier $n$, code une trajectoire de billard dans un billard carré. De telles suites sont appelées suites sturmiennes.

N. Nishioka, I. Shiokawa et J. TAmura ont donné un algorithme de construction du mot $m$ en fonction du point initial et de la direction incidente $(c f .[\mathrm{NST}])$. G. RAUZY a établi de nombreuses généralisations des suites sturmiennes : il donne, par exemple, dans un travail élaboré avec P. Arnoux ( $c f$. [AR]), une interprétation combinatoire et géométrique de certaines suites dont la complexité vaut $p(n)=2 n+1$ pour tout nombre entier $n$. P. Arnoux, C. Mauduit, I. Shiokawa et J. TAmura ont prouvé que la complexité des suites définies par des trajectoires de billard dans un cube est, pour tout $n$,

$$
p(n)=n^{2}+n+1
$$

si la direction initiale est totalement irrationnelle ( $c f$. [AMST]).

Dans ce travail, nous donnons un autre exemple de généralisation du problème du billard carré. Nous considérons le cas où le polygone est rationnel convexe. L'hypothèse de rationalité signifie que les angles entre deux côtés consécutifs sont commensurables à $\pi$. Dans ce cas, le fibré unitaire tangent se décompose en surfaces invariantes sous l'action du flot du billard. Sauf pour un nombre dénombrable de directions initiales, le flot est minimal sur ces surfaces. Nous codons les trajectoires de billard de façon naturelle, c'est-à-dire par la suite des côtés qu'elles rencontrent. Notons $k_{1} \pi / r, \ldots, k_{q} \pi / r$ les angles entre deux côtés consécutifs où $k_{1}, \ldots, k_{q}, r$ sont premiers dans leur ensemble. Pour $n$ assez grand, nous montrons que la complexité des suites obtenues est donnée par la formule suivante :

$$
p(n)=n(q-2) r+2 r .
$$

Cette expression, valable sauf pour un nombre dénombrable d'angles d'incidence, est linéaire et indépendante des conditions initiales (point de départ de la trajectoire et angle d'incidence).

TOME $123-1995-\mathrm{N}^{\circ} 2$ 


\section{Définitions, notations et résultats utiles}

\section{A. Mots infinis sur un alphabet fini.}

Soit $\mathcal{A}=\left\{a_{1}, \ldots, a_{g}\right\}$ un alphabet fini, soit $M=\left(M_{n}\right)_{n \in \mathbb{N}}$ une suite à valeurs dans $\mathcal{A}$; on dit que $M$ est un mot infini sur $\mathcal{A}$ et on le note

$$
M=M_{0} \cdots M_{n} \cdots .
$$

Soit $w=w_{0} \cdots w_{n-1}$ un mot de longueur $n$ sur l'alphabet $\mathcal{A}$; on $\operatorname{dit}$ que $w$ est un facteur de $M$ s'il existe $k$ tel que :

$$
M_{k} \cdots M_{k+n-1}=w_{0} \cdots w_{n-1} .
$$

Le langage d'ordre $n$ de $M$, noté $L_{n}(M)$, est l'ensemble des facteurs de longueur $n$ de $M$. La complexité de $M$ est l'application de $\mathbb{N}$ dans $\mathbb{N}$ qui, à un nombre entier $n$, associe le cardinal de $L_{n}(M)$. Soit $w^{\prime}$ un mot de longueur $n+1$; on dit que $w^{\prime}$ est un successeur de $w$ s'il existe une lettre $a$ telle que $w^{\prime}=w a$. Le décalage est l'application $T \operatorname{de} \mathcal{A}^{\mathbb{N}}$ dans $\mathcal{A}^{\mathbb{N}}$ qui, au mot infini $M=M_{0} \cdots M_{n} \cdots$, fait correspondre le mot infini $M^{\prime}=M_{0}^{\prime} \cdots M_{n}^{\prime} \cdots$ tel que $M_{n}^{\prime}=M_{n+1}$ pour tout nombre entier $n$.

\section{B. Billard dans un polygone.}

a) Définition $d u$ flot $d u$ billard. - (cf. [BKM].) Soit $P$ un polygone convexe du plan $\mathbb{R}^{2}$. On note $C_{1}, \ldots, C_{q}$ ses côtés orientés par les vecteurs unitaires $\vec{u}_{1}, \ldots, \vec{u}_{q}$ et $\alpha_{1}, \ldots, \alpha_{q}$ les angles entre deux côtés consécutifs. On oriente les angles à partir d'une direction donnée, par exemple $\vec{u}_{1}$. Soient $x$ un point de $P$ et $\theta$ un angle.

Règle 1. - Si $x$ est sur le bord de $P$ et n'est pas un sommet du polygone, alors $x$ appartient à un côté $C_{i}$ qui fait un angle $\beta_{i}$ avec $\vec{u}_{1}$. On identifie les couples $\left(x, \beta_{i}+\theta\right)$ et $\left(x, \beta_{i}-\theta\right)$ pour $\theta$ compris entre 0 et $\pi$.

Règle 2. - Si $x$ est un sommet de $P$, on identifie les couples $(x, \theta)$ pour tout $\theta$ appartenant à $[0,2 \pi[$.

L'espace des phases $\Omega$ est l'ensemble des couples $(x, \theta)$ pour $x$ dans $P$ et $\theta$ dans $\left[0,2 \pi\left[\right.\right.$, compte tenu des règles 1 et 2 . On définit le flot $\Phi_{t}$ du billard sur $\Omega$ de la façon suivante.

Soient $\left(x_{0}, \theta_{0}\right)$ appartenant à $\Omega$ et $t$ un nombre réel positif; le couple $\Phi_{t}\left(x_{0}, \theta_{0}\right)=\left(x_{t}, \theta_{t}\right)$ est obtenu en joignant $x_{0}$ à $x_{t}$ par une ligne polygonale $\mathcal{L}$ de longueur $t$. Chaque segment maximal de $\mathcal{L}$ a ses deux extrémités sur le bord de $P$ (sauf le premier qui débute en $x_{0}$ et le dernier qui finit en $x_{t}$ ). Sur le bord, la direction change en suivant les lois de la 
réflexion, ce qui est possible d'après la règle 1 . Si une trajectoire rencontre un sommet, elle y reste. Pour $t$ négatif, on a $\Phi_{t}\left(x_{0}, \theta_{0}\right)=\left(x_{t}, \theta_{t}\right)$ si et seulement si

$$
\Phi_{-t}\left(x_{t}, \theta_{t}+\pi\right)=\left(x_{0}, \theta_{0}+\pi\right) .
$$

En termes plus géométriques, le flot $\Phi_{t}$ est le flot géodésique sur le fibré unitaire tangent du polygone $P$ en identifiant sur le bord, les vecteurs symétriques par rapport au bord.

b) Polygone rationnel. - On dit que $P$ est un polygone rationnel si $\alpha_{1} / \pi, \ldots, \alpha_{q} / \pi$ sont des nombres rationnels. Dans ce qui suit, on écrira toujours $\alpha_{i}=k_{i} \pi / r$ avec $k_{1}, \ldots, k_{q}$ et $r$ premiers dans leur ensemble. Pour $i$ compris entre 1 et $q$, soit $\mathcal{D}_{i}$ la droite vectorielle de direction $\vec{u}_{i}$ et $S_{i}$ la symétrie par rapport à la droite $\mathcal{D}_{i}$. Notons $G_{P}$ le groupe engendré par les symétries $S_{1}, \ldots, S_{q}$. On dit que $G_{p}$ est le groupe des symétries du polygone $P$. Les éléments de $G_{P}$ agissent sur des vecteurs unitaires ou - ce qui revient au même - sur des angles orientés. Le groupe $G_{P}$ est un groupe fini de cardinal $2 r$, isomorphe au groupe diédral d'un polygone régulier à $r$ côtés $(c f .[\mathrm{CFS}])$.

Remarque. - Réciproquement, si le groupe des symétries d'un polygone convexe $P$ est fini, alors le polygone $P$ est un polygone rationnel.

c) Flot du billard dans un polygone rationnel. $-(c f .[\mathrm{ZK}]$ ou $[\mathrm{A}]$.

(i) Construction d'une surface invariante.

Fixons une direction $\varphi$. Dans un polygone rationnel, comme le groupe $G_{P}$ est fini, toute trajectoire de billard de direction initiale $\varphi$, ne prend qu'un nombre fini de directions qui sont les $\gamma(\varphi)$, pour $\gamma$ élément de $G_{p}$. D'après la structure de $G_{p}$, ce sont les directions $\varphi_{k}^{ \pm}= \pm \varphi+2 k \pi / r$, pour $k$ compris entre 0 et $(r-1)$. Si $\varphi$ n'appartient pas à $\mathbb{Z} \pi / r$, les angles $\varphi_{k}^{ \pm}$sont tous différents; c'est ce que l'on supposera dans la suite. La surface $\mathcal{M}_{\varphi}$ formée des couples $(x, \gamma(\varphi))$, pour $x$ élément de $P$ et $\gamma$ appartenant à $G_{p}$, est donc invariante sous l'action du flot du billard. La surface $\mathcal{M}_{\varphi}$ est isométrique au produit $P \times G_{p}$ quotienté par l'identification des points $(x, \gamma)$ et $\left(x, S_{i} \circ \gamma\right)$ si $x$ appartient au côté $C_{i}$.

(ii) Géométrie de la surface $\mathcal{M}_{\varphi}$.

Sous les hypothèses que nous avons faites, la surface $\mathcal{M}_{\varphi}$ est une surface compacte orientable sans bord. Elle est triangulée : elle possède $2 r$ faces isométriques à $P, q r$ arêtes, car elles sont identifiées deux à deux. On appelle $S_{i}$ ses sommets.

(iii) Exemples.

Dans les cas du carré, du triangle équilatéral, du triangle rectangle

TOME $123-1995-\mathrm{N}^{\circ} 2$ 
isocèle, la surface $\mathcal{M}_{\varphi}$ est un tore obtenu grâce au pavage du plan par ces polygones :

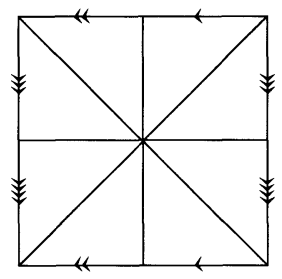

Triangle rectangle isocèle

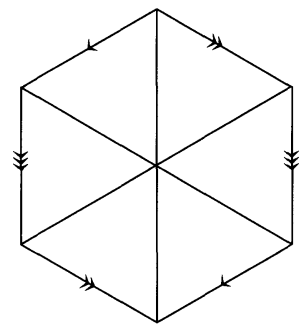

Triangle équilatéral

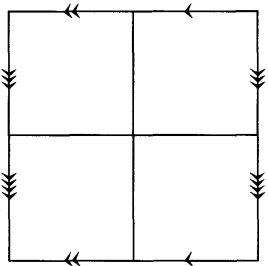

Carré

(iv) Flot du billard sur la surface $\mathcal{M}_{\varphi}$ et feuilletage.

La surface $\mathcal{M}_{\varphi}$ est munie d'une métrique plate à singularités. Les singularités sont les sommets $S_{i}$ pour lesquels l'angle au sommet est un multiple de $2 \pi$ autre que $2 \pi$. Le flot du billard définit un feuilletage mesuré singulier, orientable sur $\mathcal{M}_{\varphi}$. Les trajectoires du billard sont les feuilles du feuilletage.

$\mathrm{Au}$ voisinage d'un point régulier, le feuilletage est linéaire; localement on a la situation suivante.

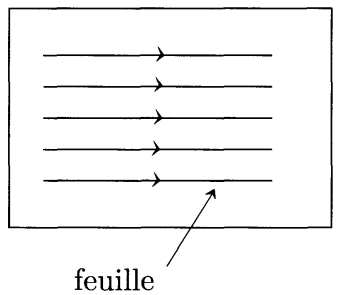

Comme le feuilletage est orientable, autour de chaque singularité il y a un nombre pair $2 p$ de branches. On trouvera deux exemples à la page suivante.

Remarque. - Dans les cas particuliers où la surface $\mathcal{M}_{\varphi}$ est un tore, le feuilletage n'a pas de singularités, le flot du billard est un flot à pente constante sur le tore. 


$$
p=2
$$

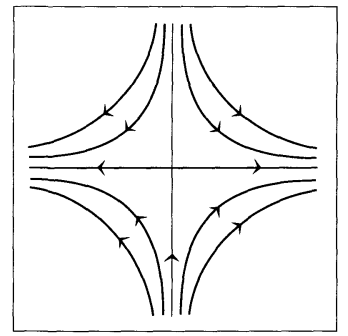

Singularité à quatre branches $p=3$

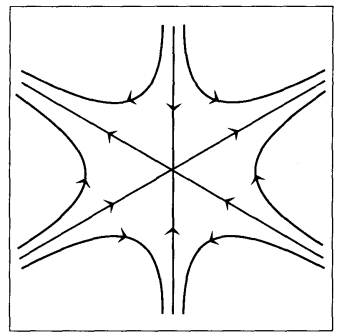

Singularité à six branches

(v) Résultat ergodique ( $c f .[\mathrm{A}])$.

DÉfinition. - On dit que $\varphi$ est $P$-rationnel s'il y a une trajectoire de billard reliant deux sommets de la surface $\mathcal{M}_{\varphi}$. Dans le cas contraire, on dit que $\varphi$ est $P$-irrationnel.

Le flot du billard sur $\mathcal{M}_{\varphi}$ est minimal si et seulement si $\varphi$ est $P$-irrationnel. Dans ce cas, vu la définition de la surface $\mathcal{M}_{\varphi}$, toute trajectoire de billard est dense dans $P \times G_{p}$. Comme l'ensemble des directions $P$-rationnelles est dénombrable, le flot du billard sur $\mathcal{M}_{\varphi}$ est minimal pour toute direction $\varphi$ sauf un nombre dénombrable.

\section{2. Énoncé du résultat}

Soient $P$ un polygone rationnel convexe, $C_{1}, \ldots, C_{q}$ ses côtés et $k_{1} \pi / r, \ldots, k_{q} \pi / r$ les angles entre deux côtés consécutifs $\left(k_{1}, \ldots, k_{q}, r\right.$ sont premiers dans leur ensemble). Soient $x_{0}$ un point du bord de $P$ et $\varphi$ un angle. On considère la trajectoire de billard du point $x_{0}$ de direction initiale $\varphi$ dans le polygone $P$. On suppose que $\varphi$ est $P$-irrationnel et que la trajectoire de $x_{0}$ ne rencontre pas de sommets du polygone. On code cette trajectoire par un mot infini $M_{0} \cdots M_{n} \cdots$ sur un alphabet à $q$ lettres $\left\{a_{1}, \ldots, a_{q}\right\}$ de façon naturelle : on considère la suite $x_{0} \cdots x_{n} \cdots$ des intersections de la trajectoires avec le bord du polygone; si $x_{n}$ appartient au côté $C_{i}$ alors $M_{n}=a_{i}$.

ThÉorème. - Soit $p(M, n)$ la complexité du mot $M$. Pour tout nombre entier $n$ assez grand, on a $p(M, n)=n(q-2) r+2 r$.

Remarque 1. - Le rang à partir duquel l'expression précédente est vraie dépend de la direction initiale $\varphi$; par contre, dès que la formule est vraie, la complexité est indépendante des conditions initiales. 


\section{EXEMPLES.}

- Dans le cas du triangle équilatéral, la complexité vaut $p(n)=3 n+6$ pour $n$ assez grand.

- Dans le cas du triangle rectangle isocèle, la complexité vaut $p(n)=$ $4 n+8$ pour $n$ assez grand.

- Dans le cas du carré, la complexité vaut $p(n)=4 n+4$ pour $n$ assez grand. On peut retrouver facilement ce résultat en utilisant le fait que si l'on code une trajectoire de billard dans un carré par la suite des horizontales et des verticales rencontrées, la complexité vaut $p(n)=n+1$ pour tout nombre entier $n$.

\section{Démonstration}

Soit $\Phi_{t}$ le flot du billard sur la surface $\mathcal{M}_{\varphi}$, notons $\Phi$ l'application de premier retour sur l'union des arêtes de la triangulation de $\mathcal{M}_{\varphi}$. Définissons un nouveau codage de trajectoires de billard. On associe à toutes les arêtes des lettres différentes. Soient $A_{i, 1}, \ldots, A_{i, r}$ les arêtes correspondant au côté $C_{i}$ du polygone $P$. On code l'arête $A_{i, j}$ par la lettre $a_{i, j}$. On construit ainsi un mot infini $M^{\prime}=M_{0}^{\prime} \cdots M_{n}^{\prime} \cdots$ qui code la trajectoire du couple $\left(x_{0}, \varphi\right)$ sur l'alphabet

$$
\left\{a_{i, j} ;(i, j) \in\{1, \ldots, q\} \times\{1, \ldots, r\}\right\}
$$

de la manière suivante : si $\Phi^{n}\left(x_{0}, \varphi\right)$ appartient à $A_{i, j}$ alors $M_{n}^{\prime}=a_{i, j}$.

Ce codage tient compte de l'angle entre les côtés du polygone et la trajectoire de billard que l'on considère.

Lemme 1. - Pour tout $n \geq 1$, on a $p\left(M^{\prime}, n\right)=n(q-2) r+2 r$.

Démonstration. - Notons $\operatorname{Tr}\left(\mathcal{M}_{\varphi}\right)$ l'union des arêtes de la triangulation de $\mathcal{M}_{\varphi}$. Soit $(x, \Psi)$ appartenant à $\operatorname{Tr}\left(\mathcal{M}_{\varphi}\right)$ et notons

$$
i(x, \Psi)=i_{0}(x, \Psi) \cdots i_{n}(x, \Psi) \cdots
$$

l'itinéraire de $(x, \Psi)$ sous $\Phi$, c'est-à-dire le codage de la trajectoire de $(x, \Psi)$ sur l'alphabet $\left\{a_{i, j} ;(i, j) \in\{1, \ldots, q\} \times\{1, \ldots, r\}\right\}$.

Remarquons que si $\Phi^{n}(x, \Psi)$ est un sommet de la triangulation, l'itinéraire de $(x, \Psi)$ n'est défini que jusqu'au rang $n-1$; c'est alors un mot de longueur $n$.

Soit $p_{1}(n)$ le cardinal de $\left\{i_{0}(x, \Psi) \cdots i_{n-1}(x, \Psi) ;(x, \Psi) \in \operatorname{Tr}\left(\mathcal{M}_{\varphi}\right)\right\}$. Il est clair que $p\left(M^{\prime}, n\right)$ est inférieur ou égal à $p_{1}(n)$. 
Montrons que $p\left(M^{\prime}, n\right)=p_{1}(n)$. Soient $(x, \Psi)$ appartenant à $\operatorname{Tr}\left(\mathcal{M}_{\varphi}\right)$ et $w=i_{0}(x, \Psi) \cdots i_{n-1}(x, \Psi)$; il existe un voisinage ouvert $\mathcal{O}$ de $(x, \Psi)$ dans $\operatorname{Tr}\left(\mathcal{M}_{\varphi}\right)$ tel que, si $\left(y, \Psi^{\prime}\right)$ appartient à $\mathcal{O}$, on ait

$$
w=i_{0}\left(y, \Psi^{\prime}\right) \cdots i_{n-1}\left(y, \Psi^{\prime}\right) .
$$

Comme, par hypothèse, la trajectoire de $\left(x_{0}, \varphi\right)$ est dense dans la surface $\mathcal{M}_{\varphi}$, il existe un nombre entier $n$ tel que $\Phi^{n}\left(x_{0}, \Psi\right)$ appartient à $\mathcal{O}$. Par conséquent, $w$ est un mot de $L\left(M^{\prime}\right)$; donc $p\left(M^{\prime}, n\right)=p_{1}(n)$.

Pour un nombre entier $n$ donné et $w=w_{0} \cdots w_{n-1}$ facteur de longueur $n$ de $M^{\prime}$, on définit

$$
C(w)=\left\{(x, \Psi) \in \operatorname{Tr}\left(\mathcal{M}_{\varphi}\right) ; i_{0}(x, \Psi)=w_{0} \cdots i_{n-1}(x, \Psi)=w_{n-1}\right\} .
$$

Comme on a codé toutes les arêtes différemment, $C(w)$ est contenu dans une seule arête $A$ de la triangulation celle codée par $w_{0}$.

Montrons que $C(w)$ est un segment de l'arête $A$. Soient $x$ et $y$ appartenant à $C(w)$. Dire que les itinéraires de $x$ et $y$ coïncident jusqu'au rang $(n-1)$ revient à dire que $\Phi^{k}([x, y])$ ne contient pas de sommet pour tout entier $k$ strictement inférieur à $n$. Par conséquent, tout élément de $[x, y]$ a le même itinéraire que $x$ jusqu'au rang $(n-1)$. Ainsi $C(w)$ est un segment.

Le mot fini $w$ a plusieurs successeurs si $\Phi^{n}(C(w))$ contient au moins un sommet $S$. Dans ce cas, on dit que $w$ se divise en $S$. Si un mot $w$ se divise en $d$ sommets, $w$ possède a priori $2 d$ successeurs mais comme $C(w)$ est connexe, il y a en seulement $d+1$ distincts.

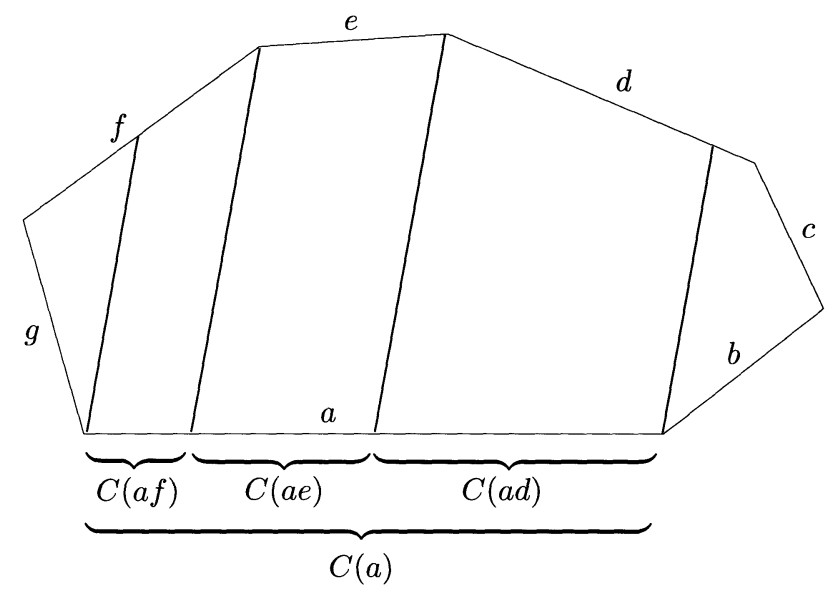

Calculer $p\left(M^{\prime}, n+1\right)-p\left(M^{\prime}, n\right)$ revient donc à déterminer combien de mots se divisent en chaque sommet.

TOME $123-1995-\mathrm{N}^{\circ} 2$ 
Soit $S$ un sommet de la triangulation. Supposons que $S$ est une singularité à $k$ branches du feuilletage associé au flot du billard sur la surface $\mathcal{M}_{\varphi}$. Comme ce feuilletage provient d'un flot, le nombre de branches de la singularité $S$ est pair : $k=2 k^{\prime}$. Par convention, si $S$ est un sommet régulier, on le considère comme une singularité à deux branches. Il y a $k^{\prime}$ trajectoires qui aboutissent en $S: \ell_{1}, \ldots, \ell_{k^{\prime}}$. Soit $x_{i}$ la $(n-1)$-ième intersection de $\ell_{i}$ avec la triangulation, lorsqu'on parcourt $\ell_{i}$ dans le sens des $t$ négatifs à partir de $S$ et $\Psi_{i}$ la direction de $\ell_{i}$ en $x_{i}$. Soit $w^{i}=i_{0}\left(x_{i}, \Psi_{i}\right) \cdots i_{n-1}\left(x_{i}, \Psi_{i}\right)$ l'itinéraire de $x_{i}$. Par construction, $\Phi^{n}\left(C\left(w^{i}\right)\right)$ contient $S$. Les mots $w^{1} \cdots w^{k^{\prime}}$ sont distincts car $w_{n-1}^{1} \cdots w_{n-1}^{k^{\prime}}$ sont différents : en effet, dans un polygone donné il y a au plus une façon d'aboutir au sommet $S$ en suivant la direction du billard.

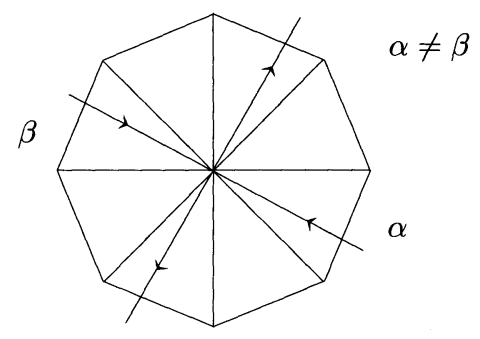

Les mots $w^{1} \cdots w^{k^{\prime}}$ se divisent au sommet $S$ et ce sont les seuls. Il y a donc $k^{\prime}$ mots qui se divisent en $S$. Par conséquent, on a

$$
p\left(M^{\prime}, n+1\right)-p\left(M^{\prime}, n\right)=\sum_{s \text { sommet }} k_{s}
$$

où $2 k_{s}$ est le nombre de branches du sommet $\mathrm{S}$. Comme la surface $M_{\varphi}$ est feuilletée, la caractéristique d'Euler de $M_{\varphi}$ est donnée par :

$$
\chi\left(M_{\varphi}\right)=\sum_{s \text { singularité }}\left(1-k_{s}\right)=\sum_{s \text { sommet }}\left(1-k_{s}\right) .
$$

Comme la surface $\mathcal{M}_{\varphi}$ est triangulée on obtient aussi la caractéristique d'Euler par :

$$
\chi\left(M_{\varphi}\right)=N(F)-N(A)+N(S)
$$

où $N(F)$ est le nombre de faces, $N(A)$ est le nombre d'arêtes et $N(S)$ est le nombre de sommets. On a donc l'égalité :

$$
\sum_{s \text { sommet }}\left(1-k_{s}\right)=N(F)-N(A)+N(S) .
$$


Donc

$$
\sum_{s \text { sommet }}\left(k_{s}\right)=N(A)-N(F)=(q-2) r
$$

et $p\left(M^{\prime}, n+1\right)-p\left(M^{\prime}, n\right)=(q-2) r$.

Comme pour $n=1$, on a $q r$ lettres : pour tout $n \geq 1$, on a

$$
p\left(M^{\prime}, n\right)=n(q-2) r+2 r
$$

Lemme 2. - Soit $w$ un mot infini sur l'alphabet

$$
\left\{a_{i, j} ; 1 \leq i \leq q, 1 \leq j \leq r\right\}
$$

qui appartient à l'adhérence de l'orbite de $M^{\prime}$ par le décalage $T$. Alors :

- soit il existe $(x, \Psi)$ appartenant à $\operatorname{Tr}\left(\mathcal{M}_{\varphi}\right)$ tel que $w$ est l'itinéraire de $(x, \Psi)$ sous $\Phi$

- soit il existe $\left(S^{\prime}, \Psi^{\prime}\right)$ appartenant à $\operatorname{Tr}\left(\mathcal{M}_{\varphi}\right)$ et un entier $k$ tel que $T^{k}(w)$ est l'itinéraire de $\left(S^{\prime}, \Psi^{\prime}\right)$.

De plus $S^{\prime}$ est l'image par $\Phi$, dans la direction $\Psi^{\prime}$, d'un sommet $S$ de la triangulation de $\mathcal{M}_{\varphi}$.

Démonstration. - Notons $\mathcal{S}=\left\{\Phi^{-k}(S)\right\}$ où $S$ est un sommet de la triangulation de $\mathcal{M}_{\varphi}$ et $k$ un nombre entier. Soit

$$
C(n, w)=\left\{(x, \Psi) \in \operatorname{Tr}\left(\mathcal{M}_{\varphi}\right) ; i_{0}(x, \Psi)=w_{0} \cdots i_{n-1}(x, \Psi)=w_{n-1}\right\}
$$

Notons $\overline{C(n, w)}$ l'adhérence de $C(n, w)$.

La suite des fermés $\overline{C(n, w)}$ est une suite décroissante pour l'inclusion; la longueur de $\overline{C(n, w)}$ tend vers zéro quand $n$ tend vers l'infini par minimalité du flot $\Phi_{t}$ sur la surface $\mathcal{M}_{\varphi}$. Donc

$$
\bigcap_{n \in \mathbb{N}} \overline{C(n, w)}
$$

est réduite à un point $Q$. On peut remarquer que les $C(n, w)$ sont des segments inclus dans une même arête de la triangulation de $\mathcal{M}_{\varphi}$. D'autre part, le bord de $C(n, w)$ est formé de deux éléments de $\mathcal{S}$. Deux cas se présentent :

TOME $123-1995-\mathrm{N}^{\circ} 2$ 
Premier cas : le point $Q$ n'appartient pas à l'ensemble $\mathcal{S}$. Pour tout nombre entier $n$, le point $Q$ appartient à $C(n, w)$. Donc

$$
\{Q\}=\bigcap_{n \in \mathbb{N}} C(n, w)
$$

et $w$ est l'itinéraire de $Q$.

Deuxième cas : le point $Q$ appartient à $\mathcal{S}$. Il existe donc un sommet $S$ et un nombre entier $k$ tel que $\Phi^{k}(Q)=S$. A partir du rang $k$, l'itinéraire de $Q$ n'est plus défini. Donc, pour tout nombre entier $n$ supérieur ou égal à $k, Q$ appartient au bord de $C(n, w)$.

Remarquons que, pour tout $n$ supérieur ou égal à $k, C(n, w)$ est d'un même côté de $Q$. On dit que $w$ est «un codage» de $Q$ en un sens que l'on va préciser. Soit $k^{\prime}$ le nombre entier supérieur à $k$ tel que $S$ appartient au bord de $\Phi^{k^{\prime}}\left(C\left(k^{\prime}, w\right)\right)$ et $S$ n'appartient pas au bord de $\Phi^{k^{\prime}+1}\left(C\left(k^{\prime}, w\right)\right)$ (voir le dessin ci-dessous). Notons $P_{k^{\prime}}$ le polygone de $\mathcal{M}_{\varphi}$ contenant $\Phi^{k^{\prime}}\left(C\left(k^{\prime}, w\right)\right)$ et $\Phi^{k^{\prime}+1}\left(C\left(k^{\prime}, w\right)\right)$.

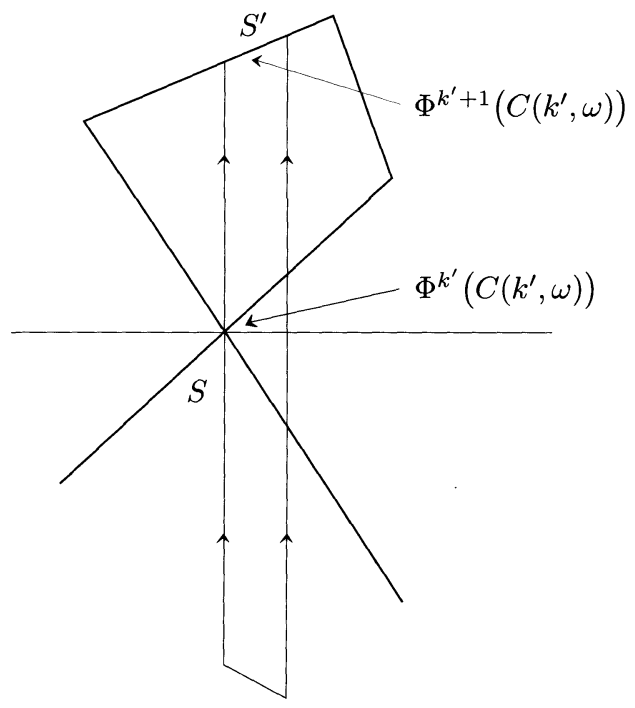

Dans le polygone $P_{k^{\prime}}$, on peut définir une trajectoire du sommet $S$. Soit $S^{\prime}$ l'image de $S$ par l'application de premier retour du flot dans $P_{k^{\prime}}$. Montrons que l'itinéraire de $S^{\prime}$ est $T^{k^{\prime}+1}(w)$ où $T$ est le décalage sur

$$
\left\{a_{i, j} ; 1 \leq i \leq q, 1 \leq j \leq r\right\}^{\mathbb{N}}
$$


Soit $n$ un nombre entier fixé : alors $\Phi^{k^{\prime}+1}\left(C\left(k^{\prime}+1+n, w\right)\right)$ est inclus dans

$$
\begin{aligned}
C^{\prime}(n, w)=\left\{(x, \Psi) \in \operatorname{Tr}\left(\mathcal{M}_{\varphi}\right)\right. & \\
i_{0}(x, \Psi) & \left.=u_{k^{\prime}+1} \cdots i_{n-1}(x, \Psi)=u_{k^{\prime}+n}\right\} .
\end{aligned}
$$

Or $S^{\prime}$ appartient au bord de $\Phi^{k^{\prime}+1}\left(C\left(k^{\prime}+1+n, w\right)\right)$, donc $S^{\prime}$ apppartient à l'adhérence de $C^{\prime}(n, w)$. Plus précisément, $S^{\prime}$ appartient à $C^{\prime}(n, w)$ sinon il y aurait une trajectoire reliant deux sommets, ce qu'on a exclu par hypothèse. Par conséquent, $S^{\prime}=\bigcap_{n \in \mathbb{N}} C^{\prime}(n, w)$ et ainsi l'itinéraire de $S^{\prime}$ est $T^{k^{\prime}+1}(w)$.

Lemme 3. - Pour $n$ assez grand, on a $p(M, n)=p\left(M^{\prime}, n\right)$.

Démonstration. - On obtient le mot $M$ à partir du mot $M^{\prime}$ par une projection lettre à lettre $p$ qui consiste à oublier l'angle entre la trajectoire et les côtés du polygone. Donc $p(M, n) \leq p\left(M^{\prime}, n\right)$ pour tout $n$.

(i) Soit $\Lambda$ l'ensemble des couples $(x, \Psi)$ appartenant à l'union des arêtes de la triangulation de $\mathcal{M}_{\varphi}$ dont l'itinéraire $i(x, \Psi)=i_{0}(x, \Psi) \cdots i_{n}(x, \Psi)$ est défini pour tout $n$. Soit $f$ l'application de $\Lambda$ dans l'ensemble des mots infinis sur l'alphabet $\left\{a_{i} ; 1 \leq j \leq q\right\}$ qui, à un couple $(x, \Psi)$, associe le mot infini $p\left(i_{0}(x, \Psi) \cdots \pi\left(i_{n}(x, \Psi)\right) \cdots\right.$.

Montrons que l'application $f$ est injective. Soient $(x, \Psi)$ et $\left(x^{\prime}, \Psi^{\prime}\right)$ éléments de $\Lambda$.

- Si $\Psi$ et $\Psi^{\prime}$ sont distincts, $f(x, \Psi)$ et $f\left(x^{\prime}, \Psi^{\prime}\right)$ sont différents d'après un argument dû à Boldrigini, M. Keane et F. Marchetti basé sur la méthode de dépliage des trajectoires ( $c f .[\mathrm{BKM}])$.

- Si $\Psi=\Psi^{\prime}$, on utilise l'hypothèse de rationalité du polygone $P$. Si $(x, \Psi)$ et $\left(x^{\prime}, \Psi\right)$ ont le même codage, par minimalité du flot $\Phi_{t}$ sur la surface $\mathcal{M}_{\varphi}, x$ et $x^{\prime}$ sont égaux.

(ii) Supposons par l'absurde que, pour tout nombre entier $n$, on ait $p(n, M)<p\left(n, M^{\prime}\right)$. En d'autres termes, il existe des mots $w^{n}=$ $w_{1}^{n} \cdots w_{n}^{n}, v^{n}=v_{1}^{n} \cdots v_{n}^{n}$ de longueur $n$, sur l'alphabet $\left\{a_{i, j} ; 1 \leq i \leq q\right.$, $1 \leq j \leq r\}$ tels que $\pi\left(w^{n}\right)=\pi\left(v^{n}\right)$. On peut supposer que les lettres $w_{1}^{n}$ et $v_{1}^{n}$ sont différentes. Par conséquent, pour tout $k$ inférieur ou égal à $n$, les mots $w_{1}^{n} \cdots w_{k}^{n}$ et $v_{1}^{n} \cdots v_{k}^{n}$ sont différents. On peut ainsi trouver deux suites de mots arbitrairement longs qui sont successeurs les uns des autres et qui vérifient les conditions précédentes. En passant à la limite, on peut donc construire sur l'alphabet $\left\{a_{i, j} ; 1 \leq i \leq q, 1 \leq j \leq r\right\}$ deux mots infinis $w, v$ qui sont distincts et vérifient $\pi(w)=\pi(v)$.

(iii) Soient $w$ et $v$ construits ci-dessus; montrons que $w$ et $v$ sont égaux. Trois cas se présentent :

TOME $123-1995-\mathrm{N}^{\circ} 2$ 
Premier cas : $w$ est l'itinéraire d'un couple $(x, \Psi)$ et $v$ est l'itinéraire de $\left(x^{\prime}, \Psi^{\prime}\right)$. Par conséquent, on a $\pi(w)=f(x, \Psi)$ et $\pi(v)=f\left(x^{\prime}, \Psi^{\prime}\right)$. Par injectivité de $f$, les couples $(x, \Psi)$ et $\left(x^{\prime}, \Psi^{\prime}\right)$ sont égaux; donc $w=v$.

Deuxième cas : il existe un entier $k$ et un sommet $S$ tel que $T^{k}(w)=$ $i\left(S^{\prime}, \Psi\right)$ où $S^{\prime}$ est l'image du sommet $S$ dans la direction $\Psi, v$ est l'itinéraire de $\left(x^{\prime}, \Psi^{\prime}\right)$. De ce fait, $i\left(S^{\prime}, \Psi\right)=i\left(\Phi^{k}\left(x^{\prime}, \Psi^{\prime}\right)\right)$. Par injectivité de $f$, $\left(S^{\prime}, \Psi\right)=\Phi^{k}\left(x^{\prime}, \Psi^{\prime}\right)$, donc $\Phi^{k-1}\left(x^{\prime}, \Psi^{\prime}\right)$ est un sommet, ce qui est exclu puisque l'itinéraire de $\left(x^{\prime}, \Psi^{\prime}\right)$ est infini.

Troisième cas : il existe un entier $k$ et un sommet $S$ tel que $T^{k}(w)=$ $i\left(S_{1}^{\prime}, \Psi\right)$ où $\mathrm{S}^{\prime}$ est l'image du sommet $S_{1}$ dans la direction $\Psi$. Il existe un entier $\ell$ et un sommet $S_{2}$ tel que $T^{\ell}(v)=i\left(S_{2}^{\prime}, \Psi^{\prime}\right)$ où $S_{2}^{\prime}$ est l'image du sommet $S_{2}$ dans la direction $\Psi^{\prime}$. Comme précédemment, $k=\ell$ et par injectivité de $f$, on en déduit $S_{1}^{\prime}=S_{2}^{\prime}$ et $\Psi=\Psi^{\prime}$. Donc $S_{1}=S_{2}=S$ et $T^{k}(w)=T^{k}(v)$. Pour vérifier que $w$ et $v$ sont égaux, il suffit de vérifier que $C(k-1, w)$ et $C(k-1, v)$ sont d'un même côté de $S$. Ceci est vérifié car sinon les lettres $\pi\left(w_{k-1}\right)$ et $\pi\left(v_{k-1}\right)$ seraient différentes.

\section{BIBLIOGRAPHIE}

[A] Arnoux (P.). - Ergodicité générique des billards polygonaux, d'après Kerckhoff, Masur, Smillie, Séminaire Bourbaki, t. 696, 1988, p. 203-221.

[AMST] Arnoux (P.), Mauduit (C.), Shiokawa (I.) and Tamura (J.). Complexity of sequences defined by billiards in the cube, Bull Soc. Math. France, t. 122, 1994, p. 1-12.

[AR] Arnoux (P.) et RAUzy (G.). - Représentation géométrique de suites de complexité $2 n+1$, Bull. Soc. Math. France, t. 119, $\mathrm{n}^{\circ} 2$, 1991, p. 199-215.

[BKM] Boldrigini (C.), Keane (M.) and Marchetti (F.). - Billiards in polygons, Annals of Probability, t. 6, 1978, p. 532-540.

[CFS] Cornfeld (I.P.), Formin (S.V.) and Sinai (Ya.G.).-Ergodic theory, Springer-Verlag, 1982.

[HM] Hedlund (G.A.) and Morse (M.). - Symbolic Dynamics II, Sturmian trajectories, Amer. J. Math., t. 62, 1940, p. 1-42. 
[K] КАток (A.). - The growth rate for the number of singular and periodic orbits for a polygonal billiard, Commun. Math. Phys., t. 111, 1987, p. 151-160.

[KMS] Kerckhoff (S.), Masur (H.) and Smillie (J.). - Ergodicity of billiard flows and quadratic differentials, Ann. of Math., t. 124, 1986, p. 293-311.

[NST] Nishioka (N.), Shiokawa (I.) and Tamura (J.). - Arithmetical properties of a certain power series, J. Number Theory, t. 42, 1992, p. $61-87$.

[ZK] ZemLyakof (A.N.) and KatoK (A.B.). - Topological transivity of billiards in polygons, Math. Notes, t. 18, $\mathrm{n}^{\mathrm{o}} 2,1976$, p. 760-764. 